\title{
CEOS Contributions to Informing Energy Management and Policy Decision Making Using Space-Based Earth Observations
}

\author{
Richard S. Eckman \\ NASA Headquarters, Mail Suite 3B74, Washington, DC USA 20546-0001 \\ Phone: +1-202-358-2567, Fax: +1-202-358-3172, Richard.S.Eckman@nasa.gov
}

\begin{abstract}
Earth observations are playing an increasingly significant role in informing decision making in the energy sector. In renewable energy applications, space-based observations now routinely augment sparse ground-based observations used as input for renewable energy resource assessment applications. As one of the nine Group on Earth Observations (GEO) societal benefit areas, the enhancement of management and policy decision making in the energy sector is receiving attention in activities conducted by the Committee on Earth Observation Satellites (CEOS). CEOS has become the "space arm" for the implementation of the Global Earth Observation System of Systems (GEOSS) vision. It is directly supporting the space-based, near-term tasks articulated in the GEO three-year work plan.

This paper describes a coordinated program of demonstration projects conducted by CEOS member agencies and partners to utilize Earth observations to enhance energy management end-user decision support systems. I discuss the importance of engagement with stakeholders and understanding their decision support needs in successfully increasing the uptake of Earth observation products for societal benefit. Several case studies are presented, demonstrating the importance of providing data sets in formats and units familiar and immediately usable by decision makers. These projects show the utility of Earth observations to enhance renewable energy resource assessment in the developing world, forecast space-weather impacts on the power grid, and improve energy efficiency in the built environment.
\end{abstract}

Keywords: Energy management, renewable energy resource assessment, Committee on Earth Observation Satellites, Group on Earth Observations, energy efficiency, decision support

\section{Introduction}

A consensus is emerging that global temperature increase from increasing greenhouse gases must be limited to $2^{\circ} \mathrm{C}$. The deployment of low carbon energy technologies and enhancing energy efficiency are key components in society's efforts to mitigate climate change impacts. [1] Earth observations are critical in enhancing the implementation of renewable energy technologies and improving energy efficiency. Measurements of solar radiation at the Earth's surface, winds, and a range of atmospheric parameters are being used to assess to potential of renewable energy resources at a given location across a variety of scales ranging from the individual household to large solar thermal power generation projects. Weather forecasts are used to optimize load forecasts by power utilities and to better integrate intermittent sources of energy into the electrical grid. Extreme weather events and climate forecasts are being used for longer-term infrastructure planning. Observations made in the near space environment assist in the prediction of potentially damaging impacts on national grids, particularly at high latitudes near the geomagnetic poles.

Recognizing the importance of Earth observations in informing decision making across a variety of disciplines - including energy, water, disasters, and air quality - the G7 countries 
called for the establishment of a Global Earth Observation System of Systems (GEOSS) at the World Summit on Sustainable Development, held in 2002. The Group on Earth Observations (GEO) is a voluntary partnership of over 80 governments and international organizations facilitating the establishment of GEOSS. A 10-year implementation plan, initiating in 2005, defines the vision for this system of systems approach.[2] Critical to the successful establishment of GEOSS is the engagement of end users who are responsible for making the management and policy decisions at national, regional, and local levels across a range of societal benefit areas. These end users are generally non-scientists, who are frequently unfamiliar with the utility of Earth observations to inform decision making. GEO emphasizes an end-to-end approach of creating data products derived from observations of the Earth system and providing them in formats and units relevant to end-user decision support systems.

Space-based observations are unique in providing a global perspective on terrestrial and nearspace processes. The Committee on Earth Observation Satellites (CEOS), an organization of 28 space agencies and 20 related national and international organizations, coordinates civil space-borne observations of the Earth. In this role, CEOS supports the space-based activities of GEO and works closely with GEO member organizations in supporting work plan tasks.

\section{Methodology}

The application of science results to inform end-user decision making requires different methodologies compared with more basic research activities. These end-users are typically non-scientists, who are unfamiliar with the science of the Earth system, conducting measurements from the ground or from space, and the intricacies of creating products that they may be used to enhance their decision support systems. GEO and CEOS seek to bridge the divide between the scientist and decision maker by conducting a variety of demonstration and prototype projects highlighting the utility of products derived from Earth observations to end-users.

Depending on the target application area, a variety of approaches are required to communicate the value of these derived, value-added products to the end-user. Several application case studies are described in the results section below, but here I describe some general approaches that have been successful for my colleagues in the GEO and CEOS energy communities of practice.

\subsection{Stakeholder Engagement and Marketing}

Scientists are typically not trained in the art of marketing. Interacting with decision makers who are frequently unaware of the utility of Earth observations requires novel methodologies. On behalf of the NASA Applied Sciences Program, a Stakeholders workshop was held in early 2007 bringing together representatives from U.S. government agencies, the power utility sector, and the renewable energy industry. [3] Our approach was to describe the availability and potential utility of space-based Earth observations and understand the needs of those making decisions relating to energy management implementation and resource assessment. My colleagues at Battelle conducted several facilitated discussions to better understand end-user requirements and to match a range of Earth observations to potential applications. The meeting was viewed as successful by the organizers and participants and led to a funded demonstration project and several ongoing partnerships. 


\subsection{Professional Societies}

An excellent venue for conveying the utility of products derived from space-based observations is at professional society meetings. My colleagues and I have engaged representatives from ASHRAE, the American Institute of Architects (AIA), and the Electric Power Research Institute (EPRI) at meetings. Researchers at the NASA Langley Research Center are working with ASHRAE committees and the U.S. Department of Energy to explore the utility of space-based observations to improve and update standards manuals related to the heating and air-conditioning industries. Discussions at AIA meetings have focused on enhancing building efficiency with products derived from Earth observations. These interactions are ongoing and have led to several projects.

\subsection{Conferences}

An essential component of our engagement is presenting research and application results at relevant science conferences. My colleagues and I have also been proactive in organizing sessions at conferences, focusing on the application of atmospheric science measurements to the energy sector. At the American Meteorological Society summer community meeting, held in August 2009, we organized a 2-day session on meeting the needs of the solar and wind energy communities from an atmospheric science perspective using Earth observations and forecasts (http://www.ametsoc.org/MEET/fainst/2009summercommunity.html).

Approximately 150 individuals from the wind and solar energy industries, academia, and government met to discuss issues relating to enhancing the uptake of atmospheric science measurements to support these industries.

\subsection{Publications}

Communicating the successes of the utility of the application of Earth observations to the energy sector requires not only the use of traditionally focused science and applications journals, books, and conference proceedings, but also more novel Web-based methods like podcasts (see, e.g., http://blogs.aip.org/CLEAN/jrse-podcast.xml), blogs (e.g., http://environmentalresearchweb.org/cws/article/futures/35553) and articles in the press (e.g., http://www.busrep.co.za/index.php?fSectionId=\&fArticleId $=4163644$ and http://www.earthzine.org/2009/07/06/from-satellite-to-solar-walls-a-look-at-geo-energy). [4, 5] Engagement of the media is often fortuitous, based on chance meetings at conferences, but increasingly depends on reporters and other interested individuals discovering our work on the Web.

\subsection{Internet Search Engines}

The importance of acquiring an Internet "footprint" cannot be overstated. As one disseminates the results of Earth observation applications on the Web, they are cataloged by the major search engine sites. Potential end-users and other interested parties can find these results while searching the Web. For example, a search using the keywords "space observations energy management" employing several of the major Internet search engines yields a wealth of relevant information on GEO and CEOS energy community of practice activities. I have received a number of enquiries relating to our renewable energy and energy efficiency products as a result of Web searches by potential end-users and correspondents. 


\section{Results}

I describe the methodology of two research groups, supporting GEO work plan tasks, which make available parameters relevant to decision makes in the energy sector. These data are used in a variety of manners by end-users. Several case studies showing the use of these data in decision support tools and systems are illustrated.

\subsection{Tailored Data Products}

Typically, those making decisions about energy utilization or deployment are resource managers, financers, and private citizens in both the developed and developing world. These individuals are generally not scientists and not familiar with the utilization of data derived from Earth observations. My colleagues at NASA and in other agencies participating in the GEO and CEOS energy communities of practice have found that data sets must be tailored to end-user requirements by packaging the products in easily usable formats, and made downloadable on the Web or through other digital data sources (e.g., data CDs and DVDs).

Researchers at the NASA Langley Research Center have created a data set formulated for assessing and designing renewable energy and energy efficient systems. [6] The Surface Meterology and Solar Energy (SSE) data set is derived from multiple space-based measurements, climatologies, and Earth system model reanalyses. Over 200 parameters relating to solar energy resource assessment, cloud cover, and meteorological conditions are available globally on a $1^{\circ} \times 1^{\circ}$ latitude/longitude grid for a 22-year period from 1983-2006. These data are made available in ASCII format and through graphical plots, enabling the nonspecialist to easily view and manipulate the data. Figure 1 shows an example of a solar energy product from the SSE data set. The long data time series allows for examination of the effects of inter-annual variability and long-term trends, possibly related to climate change impacts. The data set is freely available at the NASA Langley Atmospheric Science Data Center (http://power.larc.nasa.gov).

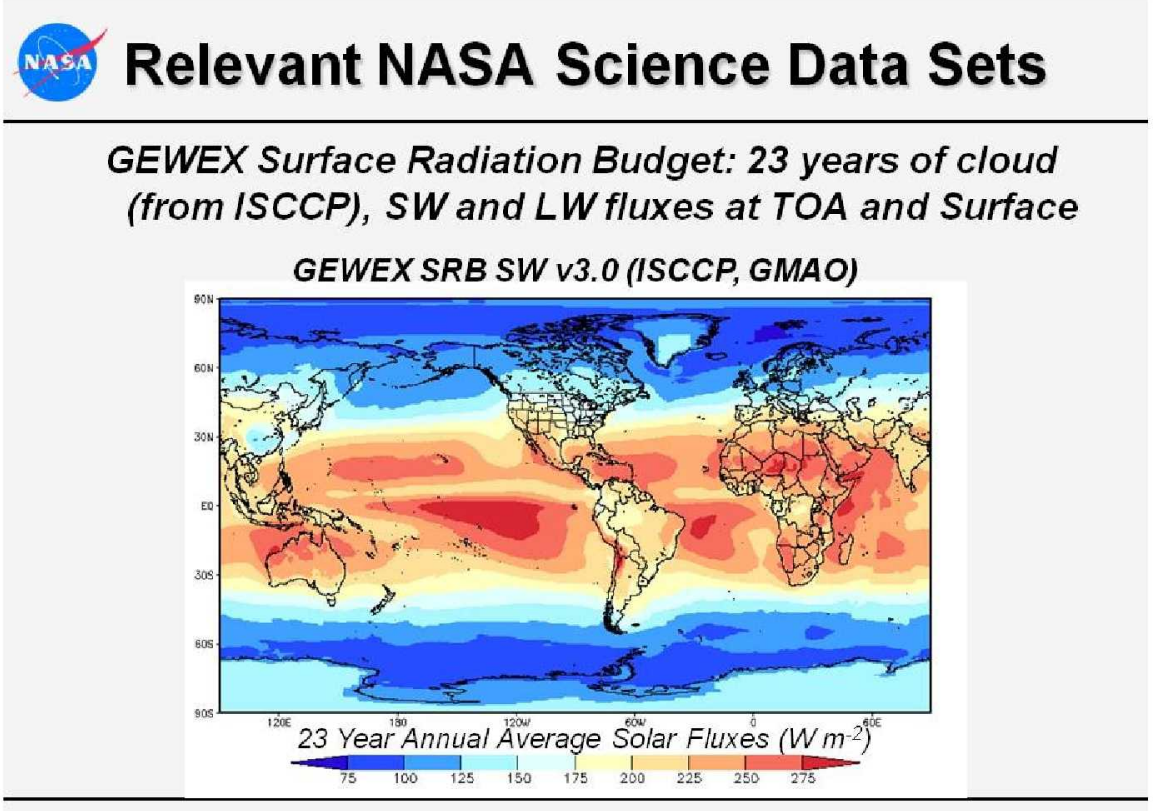


Fig. 1. An example of global solar energy products from the NASA SSE data set.

A related data set produced by the École des Mines de Paris with funding from the European Commission uses observations from geostationary meteorological satellites from Europe, USA, and Japan to produce maps of surface solar radiation. [7] These data are made available through the "SoDa" Knowledge of Solar Radiation service (http://www.soda-is.com) in both free and fee-based products. A GEO early demonstration project created a compound data base utilizing the European HelioClim and NASA SSE datasets to enable simple access to solar resource data for developing countries. This data base was demonstrated at the GEO IV Plenary in Cape Town, South Africa, in December 2007.

\subsection{RETScreen}

Natural Resources Canada's RETScreen clean energy project analysis software is an example of an end-to-end project, where data from Earth observations are directly ingested into a decision support tool (DST). NASA has partnered with Natural Resources Canada since 2000 by providing data from the SSE data set as input into the RETScreen system. The global NASA SSE data set augments input parameters in regions where ground-based data are sparse or non-existent. RETScreen's use of either ground-based or satellite-derived climate data is performed in a transparent fashion, so that the end-user is not concerned with the intricacies of importing unfamiliar data sets into the decision support system.

RETScreen, freely available on the Web at http://www.retscreen.net, has a registered user data base of nearly 225,000 users in 222 countries and is available in 26 languages. Natural Resources Canada provides manuals, video tutorials, and on-site training throughout the world. The DST is used to evaluate a wide range of renewable energy and energy efficiency technologies from a resource assessment, emission reduction, and financial viability perspective for both small-scale projects (e.g., renewable energy at the village level) to largescale power generation projects.

\subsection{HOMER}

NASA has also maintained a partnership with the U.S. National Renewable Energy Laboratory (NREL) and provided data products for use in its HOMER distributed power optimization model, available freely at https://analysis.nrel.gov/homer/. HOMER is a computer model that enables the evaluation of design options for both off-grid and gridconnected power systems for remote, stand-alone, and distributed generation applications. HOMER uses a variety of NASA data products, including the SSE data set, as meteorological and solar energy inputs to the model. HOMER has been downloaded by over 30,000 users worldwide.

\subsection{MESOR}

The "Management and Exploitation of Solar Resource Knowledge" (MESOR) project, supported by the European Commission's Sixth framework program, is designed to improve the dissemination of solar resource data by enhancing standardization and quality control (http://www.mesor.org). Links to end-users have been made via workshops, surveys, and a prototype Web portal.

\subsection{Space Weather Impacts on the Power Grid}


Solar flares can sometimes produce geomagnetic storms at high latitudes. Under some circumstances, these storms can induce geomagnetically induced currents (GICs) flowing in power transmission lines. These GICs can perturb the power grid and, if strong enough, can cause serious impacts. A large solar flare in March 1989 led to GICs which caused the collapse of the Hydro Quebec power grid for much of a day. Researchers from the NASA Goddard Space Flight Center, in collaboration with the Electric Power Research Institute (EPRI), are conducting a project to improve advance warning of the impacts of geo-effective solar flares on the power grid. [8] Observations of the sun and near-Earth space environment from platforms such as the Solar and Heliospheric Observatory and Advanced Composition Explorer are being used to improve models of the propagation of solar flares into the magnetosphere and ionosphere and the downward propagation of the impacts of geomagnetic storms to the Earth's surface. These data are ingested by EPRI's SUNBURST network for monitoring GICs and providing forecasts of impending events to the power industry.

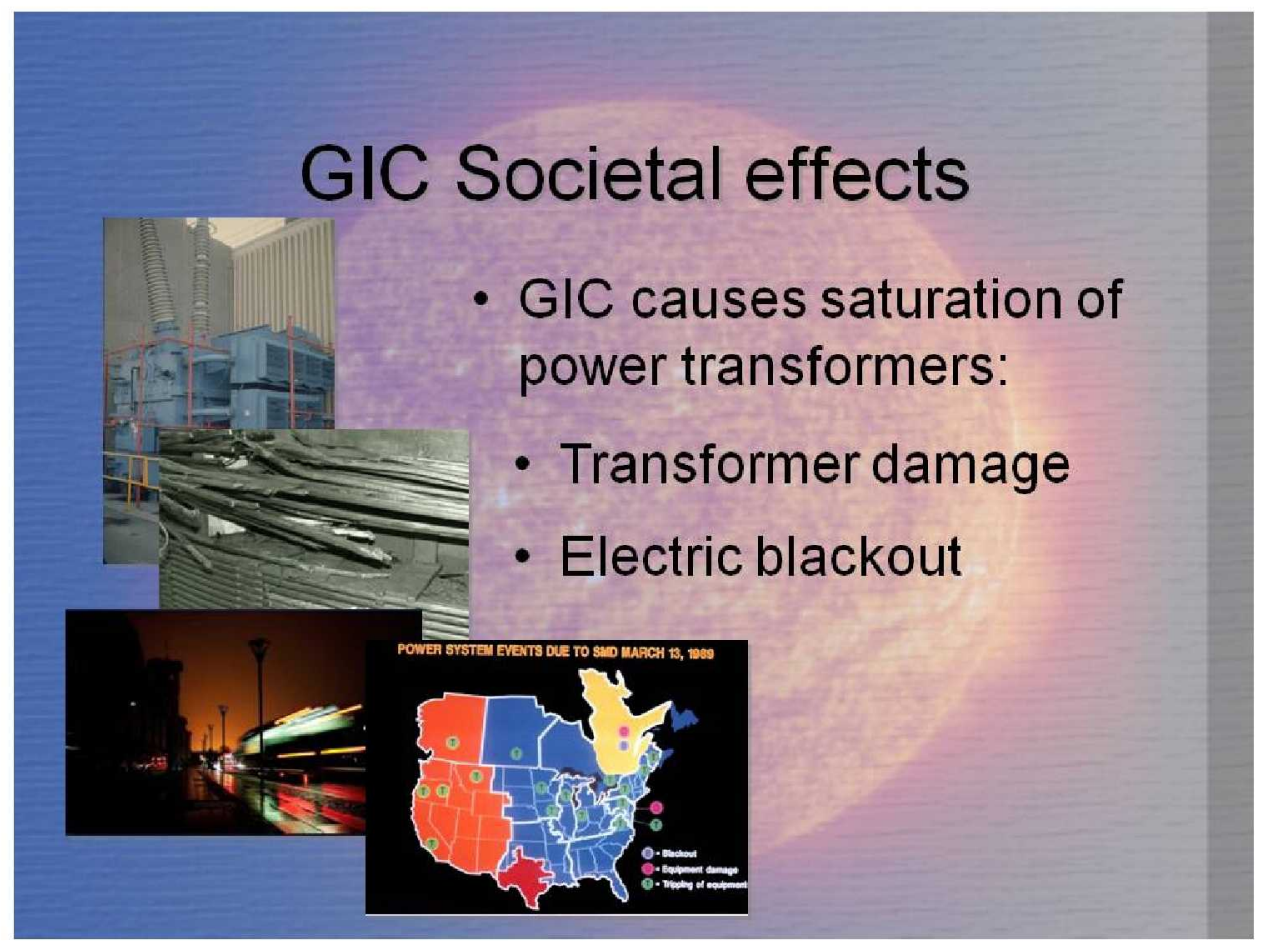

Fig. 2. Societal effects of geomagnetically induced currents (GICs) (courtesy of A. Pulkkinen, NASA).

\section{Collaborations}

\subsection{International Energy Agency}

"Solar Resource Knowledge Management" is a five-year task, established in 2005 under the International Energy Agency (IEA) Solar Heating and Cooling Programme. Coordinated by NREL, this task is an international collaboration of 8 countries and more than 15 organizations to improve solar resource standardization using space-based and surface-based data sources. The NASA SSE, DLR-led MESOR, and École des Mines' SoDA projects are among the collaborators in this IEA task, which directly supports GEO energy societal benefit area goals. In 2008, the task focused on benchmarking solar data products and on the 
development of solar forecasting methods. By 2010, a best practices guide meeting the needs of stakeholders, utilities, financial planners, and other end-users is will be published to capture the key findings of the 5-year activity. Further information on the IEA task may be found at http://www.iea-shc.org/task36/index.html.

\subsection{United Nations Environment Programme}

The United Nations Environment Programme (UNEP) Solar and Wind Energy Resource Assessment (SWERA) program began in 2001 as a pilot project to advance renewable energy technology deployment in 13 pilot countries in Asia, Africa, Central and South America. Working with NASA, NREL, and other international agencies, researchers from the United States Geological Survey (USGS) have extended these renewable energy data products globally using a variety of space-based and surface-based measurement sources including the NASA SSE data set and NASA Shuttle Radar Topography Mission measurements. These data are provided with an extensive array of geographical information system (GIS) tools to enable the end-user to map and manipulate the data. The researchers are also prototyping a complementary small hydropower assessment on the Website. This project is a CEOS contribution to the GEO work plan task relating to the management of energy sources. This newly updated global renewable energy decision support system is available freely at http://www.swera.net.

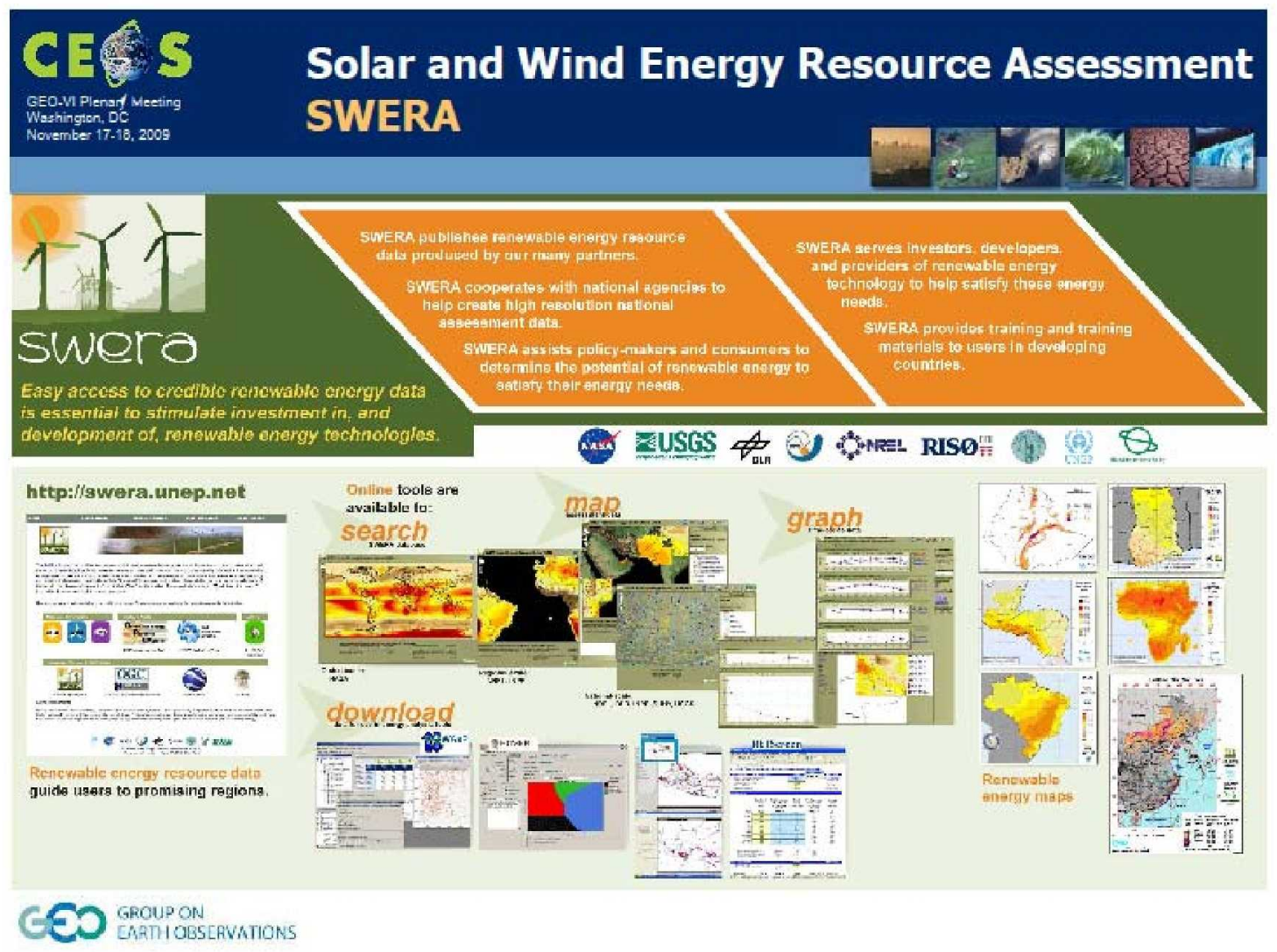

Fig. 3. An overview of the UNEP SWERA project (courtesy of Eric Wood and Gene Fosnight, USGS). 


\section{Conclusions}

I have described several case studies demonstrating the utility of data sets derived from Earth observations and Earth science model products to enhance end-user decision making in the energy sector. Working with GEO and CEOS, national space agencies, end-user organizations, and stakeholders have partnered to deliver and utilize specialized data sets to optimize and deploy renewable energy technologies, improve energy efficiency, and provide advance forecast of impending dangers to the power grid.

A key lesson learned from these early projects is the need for sustained end-user engagement. I have summarized a variety of methods used by my colleagues to engage stakeholders, provide information on the utility of Earth observations, and better understand their requirements. Successful outcomes require scientists and data owners to make these Earth observation products available in formats tailored to the needs of end-users and using Webbased and other delivery systems to disseminate these products in a timely manner.

Challenges remain in the end-to-end delivery of scientific observations to improve decision support systems. Once prototype projects are successfully demonstrated, funding must be found to maintain the delivery of data in an operational environment. Only limited progress has been made thus far in transitioning these systems to operational agencies and private industry. Non-traditional funding sources are being examined to augment government funding of these prototype projects. Nonetheless, the examples given in this paper demonstrate the promise of the utility of constructing a Global Earth Observing System of Systems for societal benefit in the energy sector.

\section{Acknowledgements}

The cases studies and methodologies described in this paper are the result of extensive experimentation by my colleagues at CEOS-member agencies and partner organizations. I gratefully acknowledge the support and advice from many of these researchers, including Paul Stackhouse and the POWER team (NASA Langley Research Center), Erica Zell and Jill Engel-Cox (Battelle), Marion Schroedter-Homscheidt (Deutsches Zentrum für Luft- und Raumfahrt), Thierry Ranchin, Lucien Wald, and Lionel Menard (École des Mines de Paris Center for Energy Processes), Ellsworth LeDrew (University of Waterloo), Fernando Ramos and Alexia Massacand (GEO Secretariat), Antti Pulkkinen (NASA Goddard Space Flight Center, and Greg Leng (RETScreen International).

\section{References}

[1] International Energy Agency, World Energy Outlook 2009, OECD/IEA, Paris, 2009, p. 45.

[2] GEO Secretariat, "The Global Earth Observing System of Systems (GEOSS) 10-Year Implementation Plan," [Online]. Available: http://www.earthobservations.org/documents/10Year\%20Implementation\%20Plan.pdf, 2007.

[3] Zell, E., and Engel-Cox, J., "NASA Energy Management Program Stakeholder Workshop: Summary Report", [Online]. Available: http://aspires.gsfc.nasa.gov/upload/EM_proj-233_5890_EM_eval_report.pdf 2007.

[4] Zell, E., Engel-Cox, J., Eckman, R., and Stackhouse, Jr., P., Application of satellite sensor data and models for energy management, IEEE Journal of Selected Topics in Applied Earth Observations and Remote Sensing, 2008, doi:10.1109/JSTARS.2008.2001142: 1-13. 
[5] Eckman, R. S., and Stackhouse, Jr., P. W., "Informing decision making in the energy sector using NASA spaceborne observations and model predictions," In: GEO Secretariat (Ed.), The Full Picture, Tudor Rose, United Kingdom, 2007, pp. 195-197.

[6] Stackhouse, Paul W., Jr., T. Zhang, W.S. Chandler, C.H. Whitlock, J.M. Hoell, D.J. Westberg, R. Perez, S. Wilcox, Satellite Based Assessment of the NSRDB Site Irradiances and Time Series from NASA and SUNY/Albany Algorithms, Proceedings of the Solar 2008 Conference (American Solar Energy Society), May 3-8, 2008, San Diego, California.

[7] Diabat, L., Blanc, Ph., and Wald, L., Solar radiation climate in Africa, Solar Energy, 2004, 76: 733-744.

[8] Pulkkinen, A., Hesse, M., Habib, S., Van der Zel, L., Damsky, B., Policelli, F., Fugate, D., and Jacobs, W., Solar Shield - forecasting and mitigating space weather effects on high-voltage power transmission systems, Natural Hazards, 2009, doi:10.1007/s11069-009-9432-x. 